\title{
Exclusão Digital em processos de Transformação Digital: uma revisão
}

\section{sistemática de literatura}

\section{Digital Divide in Digital Transformation processes: a systematic literature review}

Luzia Menegotto Frick de Moura*1, Edimara Mezzomo Luciano ${ }^{1}$, Rosiane Alves Palacios ${ }^{1}$, Guilherme Costa Wiedenhöft ${ }^{2}$

${ }^{1}$ Pontifícia Universidade Católica do Rio Grande do Sul, Rio Grande do Sul, Brasil.

${ }^{2}$ Universidade Federal do Rio Grande, Rio Grande do Sul, Brasil.

\begin{tabular}{|c|c|}
\hline I N F O A R T I GO & R E S U MO \\
\hline $\begin{array}{l}\text { Palavras-chave: } \\
\text { Transformação Digital, } \\
\text { Exclusão Digital, } \\
\text { Revisão Sistemática de } \\
\text { Literatura. }\end{array}$ & $\begin{array}{l}\text { O presente artigo tem como objetivo identificar os estudos que têm sido } \\
\text { desenvolvidos sobre exclusão digital (ED) em processos de transformação } \\
\text { digital (TD), entendendo que a tecnologia impacta a forma como as } \\
\text { sociedades e seus processos são desenvolvidos. Para isso, realizou-se uma } \\
\text { Revisão Sistemática de Literatura por meio da análise de } 21 \text { artigos, os quais } \\
\text { foram avaliados quanto à temática de estudo e à referência à exclusão digital. } \\
\text { Identificou-se uma alta taxa de utilização de surveys e análise de dados } \\
\text { secundários para a avaliação da exclusão digital, e grandes áreas de } \\
\text { concentração de temas como campo versus cidade, conflito entre gerações e } \\
\text { a questão de infraestrutura. Porém, de forma geral, ainda é baixa a } \\
\text { preocupação com a Exclusão Digital em processos de Transformação Digital. } \\
\text { Tendo isso em vista, a partir das observações feitas na Revisão Sistemática de } \\
\text { Literatura, propõe-se uma agenda de pesquisa para contribuir com o campo } \\
\text { de estudos em Exclusão Digital. }\end{array}$ \\
\hline A R T I C LE I N F O & A B S T R ACT \\
\hline $\begin{array}{l}\text { Keywords: } \\
\text { Digital Transformation, } \\
\text { Digital Divide, } \\
\text { Systematic Literature } \\
\text { Review. }\end{array}$ & $\begin{array}{l}\text { This article aims to identify the studies that have been developed on digital } \\
\text { divide (DD) in digital transformation processes (DT), focusing on the } \\
\text { understanding that technology impacts the ways societies and their } \\
\text { relationships are developed. For this, a Systematic Literature Review was } \\
\text { carried out, from which } 21 \text { articles, were extracted from databases and } \\
\text { analyzed regarding the study theme and the reference to digital exclusion. It } \\
\text { was identified a high rate of use of surveys and analysis of secondary data for } \\
\text { the evaluation of the digital exclusion, and large areas of themes concentration } \\
\text { such as countryside versus city, conflict between generations and the issue of } \\
\text { infrastructure. But, in general, the concern with Digital Divide in processes of } \\
\text { Digital Transformation is still low. Based on the observations made in the } \\
\text { Systematic Literature Review, a research agenda is proposed to contribute to } \\
\text { the field of studies in Digital Exclusion, mainly in the region of South America } \\
\text { and its specific context. }\end{array}$ \\
\hline
\end{tabular}

* Correspondência para autor:

luzia.moura@edu.pucrs.br (de Moura, L.M.F.) (ORCID: 0000-0002-4997-7985), eluciano@pucrs.br (Luciano, E.M.) (미이0002-2847-8845), rosiane.palacios@edu.pucrs.br (Palacios, R.A.) (0000-0002-7588-6358), wiedenhoft@furg.br (Wiedenhöft, G.C.) (0000-0003-2426-4396). 


\section{INTRODUÇÃO}

A humanidade e os sistemas por ela construídos, ao longo do tempo, vêm atravessando grandes transformações, sendo a tecnologia o meio para tais mudanças. Cronologicamente, tal afirmação é sustentada pela apreciação das revoluções industriais, nas quais a máquina a vapor e a nova organização do trabalho revolucionaram o modo de ser e agir dos seres humanos (SCHWAB, 2013). Mais recentemente, o mesmo ocorreu com Internet e as grandes mudanças nas esferas individual e organizacional, em virtude das novas tecnologias que permitiram o acesso ubíquo a informações, sites e aplicativos, a partir de diferentes dispositivos. Alguns exemplos mais de fronteira são a Inteligência Artificial, a Internet das Coisas, a grande massa de dados disponibilizados na rede, ensejando o surgimento do data analytics, no sentido da geração de valor e conhecimento.

Contemporaneamente, um conceito muito difundido, inclusive nas pesquisas do campo de Sistemas de Informação, é o de Transformação Digital (TD). A TD é entendida como "um processo que visa melhorar uma entidade, provocando alterações significativas em suas propriedades por meio de combinações de Tecnologias de Informação e Comunicação (TIC), computação, comunicação e conectividade" (VIAL, 2019, p. 121). Desta forma, pode-se dizer que a TD permeia as organizações, seu funcionamento e as relações interpessoais e interorganizacionais a partir da introdução de ferramentas de tecnologias digitais. A TD envolve a definição de novas estratégias, para que se possa produzir modelos de negócios e de gestão que se adaptem a essa nova realidade (REIS et al., 2018).

As tecnologias digitais permitem cada vez mais a conexão de pessoas em diferentes locais e a virtualização dos processos de trabalho (INTERNATIONAL ICT LITERACY PANEL, 2002). A rede de conexões globais possibilitada pela Internet foram fundamentais para ampliar as perspectivas econômicas (ASGARKHANI, 2005), tendo em vista o modo ubíquo de acesso à informação e a processos de interação de compra e venda. Além disso, as tecnologias foram ganhando espaço rapidamente na sociedade com a evolução dos microprocessadores, a computação em nuvem e a redução do custo dos dispositivos. Soma-se à essa isso o desenvolvimento das telecomunicações, que expandiram o acesso à Internet e assim a emergência a uma sociedade conectada. Dentre uma das características das TICs está a confluência de tecnologias em um sistema bastante integrado, tornando quase indistinguíveis as trajetórias evolutivas das inovações (CASTELLS, 1999).

Porém, à TD, ao mesmo tempo em que traz oportunidades, traz uma série de desafios. Enquanto organizações privadas possuem necessidade de adotar tecnologias para que se mantenham competitivas no mercado, organizações públicas enfrentam a urgência de entregar mais serviços na forma digitalizada (MERGEL, EDELMANN e HAUG, 2019). Isso significa que os cidadãos e empresas precisam ter acesso à tecnologia para poder utilizar estes serviços, entender como operar e, com isso, obter a informação e concluir os serviços que precisam. A falta de acesso às TICs é uma das principais formas de tornar uma família excluída dessa lógica, principalmente por não ter acesso mínimo à Internet, dispositivos de tecnologias, ou mesmo não saber utilizá-las (OCDE, 2001). Assim, os potenciais benefícios da TD se tornam sem efeito na medida em que se observam elevados níveis de exclusão digital. Outros fatores como idade, nível educacional, residir em determinada região do país, também atuam como barreiras para que as pessoas desfrutem dos potenciais benefícios da TD.

A questão da exclusão digital não é um fator tão simples como ter o acesso à Internet, mas sim uma ocorrência de problemas como a pobreza e a desigualdade (CHETTY et. al., 2018). E esse desequilíbrio é identificado na relação de forças do mercado, no investimento desigual em infraestruturas, na discriminação, em esforços políticos insuficientes, na cultura de competição. A exclusão digital se manifesta além do fato de não se possuir dispositivos de TIC ou não ter acesso a eles, mas também ao não saber usá-los (HOSMAN e COMISSO, 2020).

De forma específica, entende-se exclusão digital no contexto da transformação digital como algo que impede ou limita um indivíduo de se inserir no novo modus operandi consequência da TD, tanto em termos de vida pessoal e em comunidade como profissional, ou mesmo algo que comprometa a sua sobrevivência e o exercício de suas liberdades (SEN, 2010; CHETTY et. al., 2018). Recentemente, foi possível acompanhar os impactos negativos da exclusão digital na distribuição do auxílio emergencial à COVID19 concedido pelo governo federal para assegurar renda mínima aos cidadãos brasileiros em maior situação de vulnerabilidade social (LUCIANO, 2020). Para conseguir receber o auxílio, a população precisou fazer solicitação online, o que envolveu ter acesso à internet e cadastro em aplicativo. Porém, milhares de pessoas sequer têm Cadastro de Pessoa Física (CPF) ou mesmo um smartphone, ou o que possuem não tem capacidade para processar um aplicativo bancário. Ademais, algumas áreas do país estão fora do alcance do sinal da rede móvel e uma a cada quatro pessoas não têm acesso à internet (IBGE, 2018), levando os cidadãos a usarem um dos acessos mais caros à Internet do mundo, qual seja, as redes $3 \mathrm{G}$ ou 
$4 \mathrm{G}$.

Assim sendo, a TD tem impactado fortemente a sociedade, mudando a forma de organização e gestão de empresas e setor público, mudanças essas sentidas principalmente na implementação de novas TICs e na digitalização de serviços (MERGEL et. al., 2019). No entanto, a tecnologia, ao mesmo tempo em que dinamiza e une pessoas, provoca uma nova forma de desigualdade social, neste caso, para as pessoas que não possuem acesso à tecnologia ou que não conseguem utilizá-la. Para tanto, é importante que se estude a questão da exclusão digital visando o desenvolvimento inclusivo e próspero para todos, pois, em um mundo digital, quem tem habilidade e conhecimento para utilizar tecnologias, acaba por ter privilégios sobre quem não o tem, acentuando assimetrias entre diferentes grupos populacionais. Inclusão digital já representou um fenômeno mais contido como o uso de processadores de textos e planilhas, buscas em browsers e utilizações de e-mails (LUCIANO, 2004), mas hoje ela é social e é política, é necessária para que o cidadão tenha o pleno exercício da cidadania e das suas liberdades.

Dentro do contexto apresentado, esta pesquisa tem como foco o estudo da exclusão digital dentro dos processos de transformação digital, identificando o que esta ação gera nos indivíduos dentro dessa nova modelagem social. Percebendo um campo relativamente recente de investigação, e conforme problemática apresentada, questiona-se: O que está sendo pesquisado sobre Exclusão Digital dentro de processos de Transformação Digital?

De forma geral, o processo de TD pode se dar em diferentes frentes, podendo ser estudado tanto a partir da repaginação de modelos de negócios (por exemplo, ver SCHALLMO, WILLIAMNS, BOARDMAN, 2017) quanto em organizações públicas, com a melhoria da entrega de serviços (vide CURTIS, 2019), por exemplo. Ambas análises geram um desdobramento: a necessidade de utilização de TICs para o exercício das atividades e, como visto anteriormente, quem não conseguir manusear ou entendê-las, acaba excluído digitalmente. Assim sendo, o estudo em questão buscou identificar os temas e abordagens pesquisados no âmbito da TD que tratam sobre a exclusão digital, objetivando compreender os principais fenômenos já identificados e, com isso, contribuir com uma agenda de futuros estudos que considere o tema à luz da inclusão digital.

Seguindo as instruções de Brenton et al. (2006) para trabalhos utilizando Revisão Sistemática da Literatura (RSL), a próxima seção apresenta o método utilizado, e em seguida o desenvolvimento da RSL, os dados obtidos na seção de análise de resultados e, por fim, a conclusão do estudo.

\section{MÉTODO DE PESQUISA}

As etapas da RSL utilizadas para a realização deste trabalho tiveram como guia o trabalho de Brereton et al. (2006), que recomenda três fases principais: o planejamento (1), a condução da revisão (2) e sua documentação (3). Cada fase é composta pelos seguintes desdobramentos: (1) questão de pesquisa específica, protocolo de revisão e sua validação; (2) identificação, seleção, avaliação, coleta de dados e sua síntese; (3) escrita e validação da pesquisa. $\mathrm{O}$ processo de RSL efetivado neste trabalho está descrito no Quadro 1. Sendo assim, a seção correspondente à essa parte trará, também, a base de conhecimento que foi utilizada para reflexão sobre os achados.

Quadro 1 - Processo da Revisão Sistemática da Literatura.

\begin{tabular}{|l|l|}
\hline \multicolumn{1}{|c|}{ Fase - Etapas } & \multicolumn{1}{c|}{ Procedimento } \\
\hline $\begin{array}{l}\text { 1 - Planejamento - Questão de Pesquisa, } \\
\text { Revisão do Protocolo }\end{array}$ & $\begin{array}{l}\text { Questão principal: "O que está sendo pesquisado sobre Transformação Digital } \\
\text { que se relacione ao conceito de Exclusão Digital?" }\end{array}$ \\
\hline $\begin{array}{l}2 \text { - Condução - Identificação, seleção, } \\
\text { avaliação, coleta de dados e síntese }\end{array}$ & $\begin{array}{l}\text { Foi utilizada a string de busca "digital transformation" AND “digital divide” nas } \\
\text { plataformas escolhidas. Também foi testada a string "transformação digital” E } \\
\text { "exclusão digital”, porém não foram obtidos resultados. Posteriormente, foram } \\
\text { aplicados os critérios de exclusão para a seleção dos artigos relevantes. Ao final, } \\
\text { restaram 21 artigos, cujos resultados foram sintetizados e analisados. }\end{array}$ \\
\hline $\begin{array}{l}\text { 3 - Documentação - relatório de revisão, } \\
\text { validação do estudo }\end{array}$ & $\begin{array}{l}\text { Os dados coletados foram sintetizados e documentados a partir da utilização do } \\
\text { software NVivo com o objetivo de identificar a abordagem da exclusão digital } \\
\text { nos estudos selecionados. }\end{array}$ \\
\hline
\end{tabular}

Fonte: Elaborada pelos autores. 
A partir da validação da questão de pesquisa, a próxima etapa executada foi a busca da literatura nas bases de dados Scopus, Web of Science, Sage, Science Direct, Scielo e ProQuest. A string de busca definida foi "digital transformation" e "digital divide". O termo de busca para exclusão digital foi definido a partir de leituras prévias de textos sobre o assunto. Também foi escolhido realizar a pesquisa com o termo em inglês pois, em uma busca exploratória inicial incluindo o português, não foram obtidos retornos qualificados. A quantidade de artigos em cada base está disposta na Tabela 1.

Para realizar a seleção primária dos artigos, foram escolhidos os seguintes critérios: (i) exclusão de artigos repetidos; (ii) somente artigos em inglês, contudo, também foram aceitos artigos em português e espanhol que acabaram retornando nas pesquisas por terem resumo em inglês; (iii) artigos científicos e conference papers, excluindo documentos como teses, capítulo de livros, editoriais; (iv) leitura do resumo dos artigos buscando as palavras digital transformation e digital divide, sendo obrigatória a presença da segunda no resumo ou outra de semelhança semântica, como, por exemplo inequality ou exclusion. Estes termos não foram escolhidos para a string por não ser o termo oficial que este tema é apresentado na literatura.

Dos 349 artigos coletados, após aplicados os critérios de exclusão acima citados, restaram 44 artigos para serem analisados com maior profundidade, nos quais foram explorados o que estava sendo pesquisado sobre transformação digital e sua relação com a exclusão digital. Para a seleção final, o tema da exclusão digital deveria ser apresentado com ênfase, participando do motivo principal da pesquisa. Artigos que apenas citavam a palavra digital divide, mas não exploravam sua problemática foram descartados. Toda a etapa foi realizada no Computer Assisted Qualitative Data Analysis (CAQDA) NVivo. No CAQDA foram inseridos os seguintes dados: título, ano, questão de pesquisa, definição de exclusão digital e método de pesquisa. Ao final desta fase, restaram 21 artigos, que tiveram os dados coletados e sintetizados. A próxima seção apresentará os resultados detalhados dos materiais selecionados. Os resultados detalhados estão apresentados na Tabela 1.

Tabela 1 - Processo de seleção dos artigos para RSL, espeficicando a etapa e o número de artigos que restaram em cada etapa.

\begin{tabular}{cc}
\hline Etapa (número de artigos excluídos) & Número de artigos que restaram \\
\hline Busca nas bases de dados & 349 \\
Exclusão de artigos repetidos $(\mathrm{n}=15)$ & 334 \\
Eliminação dos artigos pelos critérios de exclusão $(\mathrm{n}=290)$ & 44 \\
Remoção dos artigos que não tratavam sobre exclusão & 21 \\
digital como ponto central (n=23) & \\
\hline
\end{tabular}

Fonte: Dados da pesquisa.

A seção seguinte apresenta a discussão dos resultados identificados na análise das publicações selecionadas.

\section{ANÁLISE DOS RESULTADOS}

A primeira abordagem buscou identificar a ocorrência de termos associados ao assunto. As principais palavras são: internet, digital, government, seguidas por um grupo de seis palavras - development, divide, access, social, rural, information. Assim, podemos partir da premissa que os artigos selecionados apresentam a temática em questão a partir das palavras "digital" e "divide", mas também apresentam pontos importantes como a influência da Internet, do desenvolvimento, da importância do fator humano (people) e do destaque que o tema tem no meio rural. A Figura 1 mostra as palavras mais citadas com mais de cinco caracteres nos 21 artigos analisados. 
Figura 1 - Nuvem de palavras com as 50 expressões mais citadas nos artigos analisados na RSL.

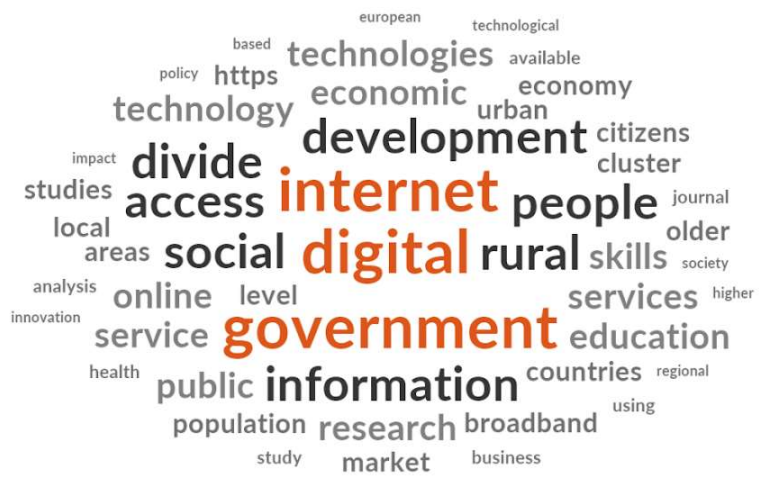

Fonte: Dados da pesquisa

Pode-se perceber que o estudo da exclusão digital dentro do campo da transformação digital é recente, pois a primeira publicação localizada foi do ano de 2014, muito embora o assunto específico da exclusão digital seja estudado desde o final dos anos 90. Este tema ganhou maior destaque nos últimos quatro anos. O ano de 2020, por exemplo, apresentou um número elevado de estudos e supõe-se que isto ocorreu justamente por ser o ano em que as TICs foram as ferramentas necessárias para garantir o trabalho remoto e a realização de serviços básicos à população em meio à pandemia global de COVID19, a qual necessita do distanciamento social como uma das formas de controle de disseminação do vírus (AYRES, 2020). O Figura 2 apresenta o compilado desses achados.

Figura 2 - Número de trabalhos por ano de publicação.

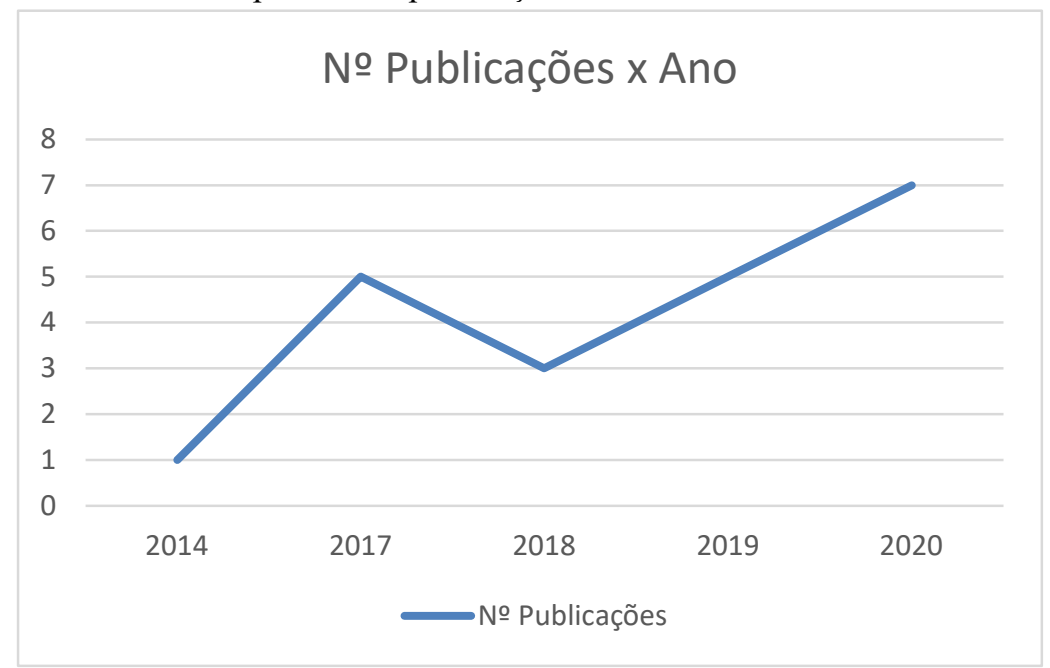

Fonte: Dados da pesquisa.

Os trabalhos selecionados apresentaram contextos de estudo tanto em países desenvolvidos quanto em desenvolvimento, o que demonstra que a exclusão digital é um tema presente em diferentes realidades econômicas e sociais. A Figura 3 apresenta os países em que esses estudos foram feitos. Nos países desenvolvidos, o principal problema identificado foi na questão de adaptação das gerações mais velhas ao uso das tecnologias, sendo seu processo de letramento digital mais complexo; também, foi observado que imigrantes eram mais vulneráveis e isolados da transformação digital. Nos países em desenvolvimento o principal problema identificado foi a questão de infraestrutura, a qual era precária para muitos habitantes. 
Figura 3 - Países identificados nas publicações na temática em estudo.

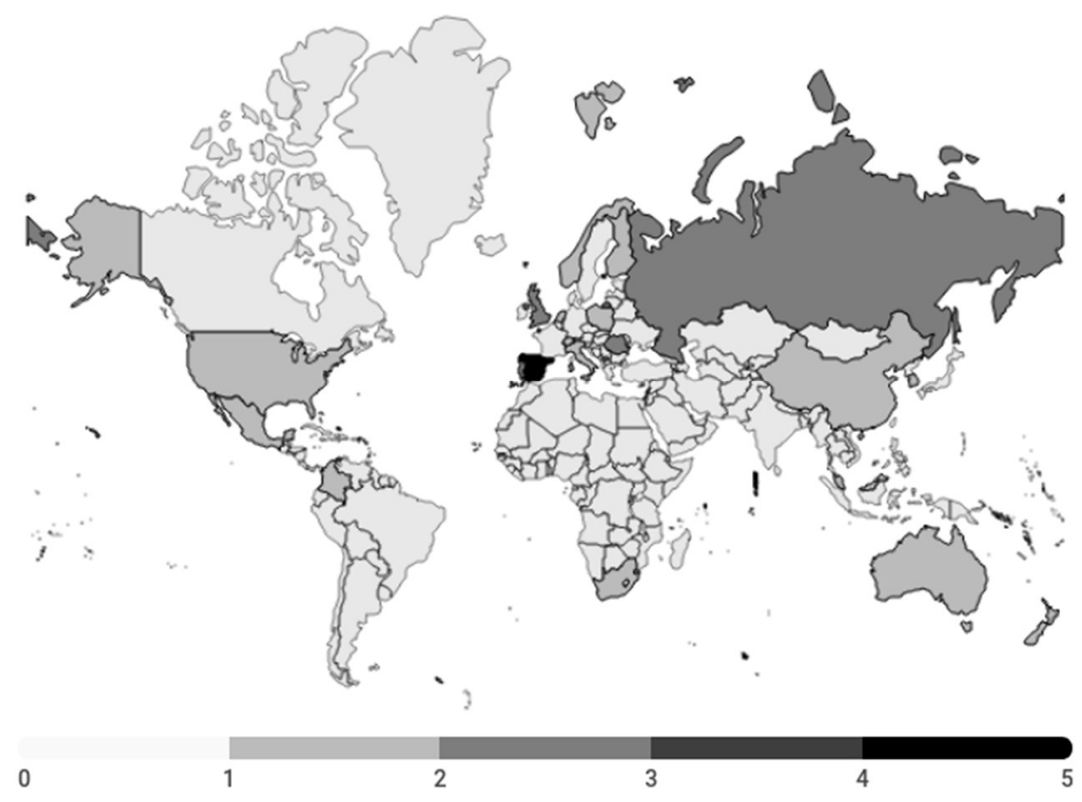

Nota: As áreas mais escuras do gráfico identificam os locais com maior produção científica sobre o tema identificados nesta RSL. Alguns artigos foram feitos em parceria entre países, podendo ser o número de países identificados maior do que a produção científica em si. Países e número de artigos: África do Sul (1), Austrália (1), China (1), Colômbia (1), Coreia do Sul (1), Espanha (5), EUA (1), Finlândia (1), Holanda (1), Itália (1), Malásia (1), México (1), Noruega (1), Nova Zelândia (1), Polônia (1), Portugal (2), Reino Unido (2), Romênia (2), Rússia (2), Suíça (1), Tonga (1).

Fonte: Dados da pesquisa.

Muitos dos artigos analisados utilizaram dados secundários, ou seja, aproveitaram surveys e outros indicadores coletados, principalmente, por fontes governamentais e também por relatórios de organizações internacionais para identificar níveis e mensurar a exclusão digital. Isso demonstra, em primeiro lugar, a importância de pesquisas lideradas pelo Estado e outras organizações com trabalhos internacionais ou regonais na formação de uma base de dados para pesquisas acadêmicas. Além disso, a busca empírica para estudar um tema, demonstra uma área nova de estudo, sendo os principais métodos de estudo o estudo de caso e a survey. O Quadro 2 resume os principais métodos identificados nos 21 artigos analisados.

Quadro 2 - Principais métodos de pesquisa utilizados

\begin{tabular}{|c|l|}
\hline Método & \multicolumn{1}{|c|}{ Referência } \\
\hline $\begin{array}{c}\text { Análise de Dados } \\
\text { Secundários }\end{array}$ & $\begin{array}{l}\text { (Domínguez, 2018), (Vasilescu, Serban, Dimian, Id, Picatoste, 2020), (Bok e Porumbescu, } \\
\text { 2019), (Zemtsov, 2020), (Otioma, Mafalda, Martinez, 2019), (Mendoza-ruano e Caldera- } \\
\text { serrano, 2014), (Aleksandrovna, Iurievna, Olegovna, 2017), (Zuhairy, Tajuddin, Rohman, } \\
\text { 2018), (Viñarás-abad, Abad-alcalá, Llorente-barroso, Sánchez-valle, Pretel-jiménez, 2017), } \\
\text { (Popova et. al., 2020), (Evans e Gomes, 2017) }\end{array}$ \\
\hline Análise bibliográfica & $\begin{array}{l}\text { (Viñarás-abad, Abad-alcalá, Llorente-barroso, Sánchez-valle, Pretel-jiménez, 2017), } \\
\text { (Popova et. al., 2020) }\end{array}$ \\
\hline Estudo de caso & (Philip e Williams, 2019), (Otioma, Mafalda, Martinez, 2019), (Gann, 2019), (Hodge, \\
\hline
\end{tabular}




\begin{tabular}{|c|l|}
\hline & Carson, Carson, Newman, Garrett, 2017), (Otioma, Mafalda, Martinez, 2019) \\
\hline Entrevistas & (Miklian e Hoelscher, 2017), (Aleksandrovna, Iurievna, Olegovna, 2017) \\
\hline
\end{tabular}

Fonte: Elaborada pelos autores

O estudo sobre Transformação Digital ainda dispõe de uma longa agenda de debate e entendimentos sobre o tema. Identificada primeiramente no setor privado como uma adaptação às novas tecnologias e dinâmica de mercado e, posteriormente, fazendo-se presente no setor público e alterando as formas de organização das instituições, a TD encontra-se intimamente ligada com a tecnologia e com as mudanças socioculturais atuais (MERGEL et. al, 2019; GONG et. al, 2020). De forma geral, apesar de possuir no seu enredo uma narrativa de mudanças positivas, a TD possui impactos negativos, mesmo que em forma de unintended consequences (VIALE PEREIRA et al., 2019). Ou seja, impactos que geralmente são imperceptíveis numa primeira análise, mas que acabam emergindo a partir do aumento das desigualdades e de outros fatores inseridos no contexto de exclusão digital global e de falta de entendimento sobre as tecnologias (PEREIRA et al., 2020).

A exclusão digital, primeiramente identificada no contexto dos EUA por ter sido o país pioneiro na ampla difusão de tecnologias e rede de Internet (SERVON, 2002), é um fator negativo a ser minimizado conforme apontado pelo nono Objetivo de Desenvolvimento Sustentável (ODS) da Organização das Nações Unidas (ONU): "Aumentar significativamente o acesso às tecnologias de informação e comunicação e se empenhar para oferecer acesso universal e a preços acessíveis à internet nos países menos desenvolvidos, até 2020" (ONU, 2020, https://brasil.un.org/pt-br/sdgs/9).

Apesar do objetivo acima exposto, ainda há muitas pessoas que não estão conectadas, e muitas das que possuem acesso à rede, não se sabe ao certo o quanto são capazes de utilizar desta (HOSMAN e COMISSO, 2020). Segundo a Organização para Cooperação e Desenvolvimento Econômico (OCDE), a exclusão digital:

Refere-se à lacuna entre indivíduos, famílias, empresas e áreas geográficas em diferentes níveis socioeconômicos, tanto no que diz respeito às oportunidades de acessar tecnologias de informação e comunicação (TICs) quanto ao uso da Internet para uma ampla variedade de atividades. $\mathrm{O}$ fosso digital reflete várias diferenças entre e dentro dos países. A capacidade de indivíduos e empresas de tirar proveito da Internet varia significativamente na área da OCDE, bem como entre a OCDE e os países terceiros (OCDE, 2001, p. 5).

Sendo assim, pode-se entender que a exclusão digital se encontra tanto na questão de infraestrutura quanto na questão de posse, acesso e utilização/entendimento das TICs. Superar essas questões torna-se uma questão de autonomia, dentro da perspectiva do desenvolvimento como liberdade (SEN, 1999).

Posto isso, buscou-se em cada artigo o conceito utilizado para a definição de exclusão digital. Apesar da apresentação do conceito variar conforme os trabalhos, todos identificaram que um dos fatores principais da exclusão digital estava principalmente na dificuldade ou na falta de infraestrutura para garantir o acesso à Internet, fatores que levam o indivíduo a ter algum tipo de prejuízo. Por outro lado, outros trabalhos traziam um segundo nível de exclusão, apresentando como influenciadores dessa desigualdade os fatores de idade, escolaridade, localidade, gênero. Isso demonstra que desigualdades já percebidas socialmente também são mantidas com a transformação digital (SERVON, 2002; CHETTY et. al., 2018). O Quadro 3 apresenta o resumo das principais expressões abordadas quanto à exclusão digital.

Quadro 3 - Principais definições de exclusão digital identificadas nos artigos estudados.

\begin{tabular}{|l|l|}
\hline \multicolumn{1}{|c|}{ Referência } & \multicolumn{1}{c|}{ Identificação da Exclusão Digital } \\
\hline Domínguez, 2018 & $\begin{array}{l}\text { A exclusão conversa com a motivação para as novas tecnologias, com o acesso } \\
\text { físico, com a alfabetização digital e com oportunidades de uso para as TICs. }\end{array}$ \\
\hline $\begin{array}{l}\text { Latapu, Lutui, Vaipuna, Moala, Fonua, } \\
\text { Liu, Gutierrez, Song, Chiaraviglio, } \\
\text { Wu, Bagula, 2018 }\end{array}$ & $\begin{array}{l}\text { A exclusão digital se refere à desigualdade entre os que conseguem acessar e } \\
\text { utilizar as TICs de quem não consegue. }\end{array}$ \\
\hline Aleksandrovna, Iurievna, Olegovna, & Diferentes níveis de acesso às tecnologias digitais, sendo analisado as diferenças \\
\hline
\end{tabular}




\begin{tabular}{|c|c|}
\hline 2017 & entre gerações dentro da mesma sociedade. \\
\hline Kupriyanova, Dronov, Gordova, 2019 & Acesso desigual às TICs \\
\hline $\begin{array}{l}\text { Vasilescu, Serban, Dimian, Id, } \\
\text { Picatoste, } 2020\end{array}$ & $\begin{array}{l}\text { Exclusão digital como falta de acesso às TICs considerando: infraestrutura, } \\
\text { escolaridade, nível de renda e outras divisões sociais (gênero, idade, etnia) }\end{array}$ \\
\hline Räisänen e Tuovinen, 2020 & Desigualdade de infraestrutura e serviços digitais nas localidades rurais. \\
\hline $\begin{array}{l}\text { Viñarás-abad, Abad-alcalá, Llorente- } \\
\text { barroso, Sánchez-valle, Pretel- } \\
\text { jiménez, } 2017\end{array}$ & $\begin{array}{l}\text { Três níveis de exclusão digital: (i) falta de acesso à Internet, (ii) capacidade de } \\
\text { utilizar as TICs e (iii) possibilidade de se conectar e utilizar as funcionalidades } \\
\text { disponibilizadas pela sociedade da informação (capacidade cognitiva). }\end{array}$ \\
\hline Bok e Porumbescu, 2019 & $\begin{array}{l}\text { Além de falta de acesso à Internet, a exclusão digital também tem relação com a } \\
\text { habilidade que o cidadão tem em utilizar as TICs. }\end{array}$ \\
\hline Zemtsov, 2020 & $\begin{array}{l}\text { Desigualdade no acesso à Internet, habilidades digitais e retornos reais advindos } \\
\text { do uso da Internet (resultados, mudança). Durante a crise desencadeada pelo } \\
\text { COVID19 essas diferenças ficaram mais pronunciadas. }\end{array}$ \\
\hline Philip e Williams, 2019 & $\begin{array}{l}\text { Primeiro nível de exclusão digital: falta de acesso à rede; segundo nível de } \\
\text { exclusão digital: diferença nas habilidades e no uso das TICs - mix de } \\
\text { desigualdades }\end{array}$ \\
\hline Miklian e Hoelscher, 2017 & $\begin{array}{l}\text { A exclusão digital apresenta lacunas que perpassam fatores como analfabetismo, } \\
\text { pobreza e baixa infraestrutura. }\end{array}$ \\
\hline Otioma, Mafalda, Martinez, 2019 & Exclusão digital quanto ao acesso e uso das TICs. \\
\hline Szeles e Simionescu, 2020 & $\begin{array}{l}\text { Trazem a exclusão digital regional, que trata sobre as desigualdades de acesso } \\
\text { dentro da região e, também, em relação à questão socioeconômica. }\end{array}$ \\
\hline Pérez-morote e Pontones-rosa, 2020 & $\begin{array}{l}\text { Acesso desigual às TICs pelos cidadãos e pouco conhecimento e habilidade de uso } \\
\text { dessas tecnologias. }\end{array}$ \\
\hline Zuhairy, Tajuddin, Rohman, 2018 & Acesso à internet. \\
\hline Popova et. al., 2020 & $\begin{array}{l}\text { A desigualdade digital é agrupada em: técnica, econômica e social. Esses grupos } \\
\text { não são isolados, são relacionados e se complementam. }\end{array}$ \\
\hline $\begin{array}{l}\text { Hayet-Kerras, Sánchez-navarro, } \\
\text { López-becerra, Gómez, } 2020\end{array}$ & $\begin{array}{l}\text { Desigualdades no acesso, uso e apropriação das TICs advindas da inovação } \\
\text { tecnológica. Quanto à exclusão digital de gênero, é a desigualdade na } \\
\text { acessibilidade às TICs quando comparado homens e mulheres. }\end{array}$ \\
\hline Evans e Gomes, 2017 & $\begin{array}{l}\text { Incapacidade de alguns segmentos da população de se beneficiar com as TICs para } \\
\text { melhoria do trabalho, escolaridade, bem-estar em geral. }\end{array}$ \\
\hline Gann, 2019 & $\begin{array}{l}\text { Dificuldades de uso e acesso aos recursos digitais, que está próximo da privação e } \\
\text { desigualdades na saúde. }\end{array}$ \\
\hline $\begin{array}{l}\text { Mendoza-ruano e Caldera-serrano, } \\
2014\end{array}$ & $\begin{array}{l}\text { Desigualdades existentes entre pessoas, comunicações ou países que possuem } \\
\text { acesso às TICs e as utilizam (principalmente internet) em relação às que não tem } \\
\text { acesso e, caso tenham, não sabem utilizá-las adequadamente. A exclusão digital é } \\
\text { uma nova dimensão que perpetua relações de poder associados a fatores } \\
\text { econômicos, raciais, culturais, geográficos, educativos e de gênero e idade. }\end{array}$ \\
\hline $\begin{array}{l}\text { Hodge, Carson, Carson, Newman, } \\
\text { Garrett, } 2017\end{array}$ & Acesso à internet e baixo conhecimento para operar a tecnologia. \\
\hline
\end{tabular}

Fonte: Dados da pesquisa.

A urbanização e suas contradições foram um tema recorrente nos trabalhos selecionados. Alguns artigos apresentaram que áreas rurais sofrem mais com a desigualdade ao acesso às TICs, muitas vezes por falta de infraestrutura, mas também por menor acesso à escola e a alfabetização, o que prejudica o correto entendimento do uso das tecnologias (Philip e Williams, 2019; Popova et. al., 2020). Isso evidencia que o meio rural ainda está afastado das inovações tecnológicas em muitos locais e políticas públicas devem ser pensadas para a inclusão 
dessas pessoas, ampliando as políticas de transformação digital para além dos grandes centros urbanos de forma positiva. Mesmo assim, alguns estudos já identificaram projetos na linha da mitigação dessas desigualdades (Räisänen e Tuovinen, 2020 Kupriyanova, Dronov, Gordova, 2019). Também foi apontada a exclusão digital por gênero, na qual foi verificado que as mulheres possuem menor acesso à tecnologia, mesmo sendo as que possuem maior facilidade de aprendizagem (HAYET-KERRAS et. al., 2020). Essa desigualdade, como é de amplo conhecimento, também está presente em outros campos da vida feminina na sociedade, como, por exemplo, a diferença salarial e a jornada dupla de trabalho (profissional e doméstica). O Quadro 4 apresenta os principais temas abordados e seus respectivos autores.

Quadro 4 - Principais temas abordados nos artigos selecionados.

\begin{tabular}{|c|c|}
\hline Temas abordados nos artigos & Referência \\
\hline $\begin{array}{l}\text { Investigar e analisar a brecha digital no México através da identificação dos fatores que } \\
\text { influenciam no acesso e uso das TICs }\end{array}$ & Domínguez, 2018 \\
\hline $\begin{array}{l}\text { Identificar como está a conectividade, o estado atual da exclusão digital e quais as possíveis } \\
\text { soluções para reduzir essa desigualdade em Tonga. }\end{array}$ & Latapu et. al, 2018 \\
\hline $\begin{array}{l}\text { Avaliar a influência do processo de transformação digital nas dinâmicas geracionais na } \\
\text { sociedade da informação }\end{array}$ & $\begin{array}{l}\text { Aleksandrovna, Iurievna, } \\
\text { Olegovna, } 2017\end{array}$ \\
\hline $\begin{array}{l}\text { Avaliar como melhorar o processo de decisão estratégica para auxiliar o desenvolvimento } \\
\text { socioeconômico dos territórios rurais e do setor agrário. }\end{array}$ & $\begin{array}{l}\text { Kupriyanova, Dronov, } \\
\text { Gordova, } 2019\end{array}$ \\
\hline $\begin{array}{l}\text { Analisar a percepção dos cidadãos da União Europeia quanto a digitalização, destacando as } \\
\text { diferenças socioeconômicas. Também, se a exclusão digital cria grupos vulneráveis. }\end{array}$ & Vasilescu et. al, 2020 \\
\hline $\begin{array}{l}\text { Demonstrar como workshops podem ser utilizados como suporte para difundir as inovações } \\
\text { digitais para microempresas rurais. }\end{array}$ & Räisänen e Tuovinen, 2020 \\
\hline Apresentar a condição que se encontra a questão dos idosos com a e-administração. & Viñarás-abad, et al, 2017 \\
\hline $\begin{array}{l}\text { Identificar se a participação do cidadão em programas governamentais de treinamento em } \\
\text { TI está associada a um maior uso de serviços de governo eletrônico entre grupos de cidadãos } \\
\text { que normalmente usam menos o governo eletrônico. }\end{array}$ & Bok e Porumbescu, 2019 \\
\hline $\begin{array}{l}\text { Intensificar a discussão sobre os impactos da digitalização e automação nos países em } \\
\text { desenvolvimento. }\end{array}$ & Zemtsov, 2020 \\
\hline $\begin{array}{l}\text { Estudar as consequências da instalação de banda larga via satélite em residências de uma } \\
\text { comunidade rural com pouco acesso à tecnologia. }\end{array}$ & Philip e Williams, 2019 \\
\hline $\begin{array}{l}\text { Assumindo as contradições que surgem com a transformação digital, buscaram propor } \\
\text { sugestões que auxiliem no crescimento urbano mais positivo e atento às necessidades dos } \\
\text { cidadãos. }\end{array}$ & Miklian e Hoelscher, 2017 \\
\hline Entender a exclusão digital no meio urbano, a partir do acesso às TICs disponíveis. & $\begin{array}{l}\text { Otioma, Mafalda, Martinez, } \\
2019\end{array}$ \\
\hline $\begin{array}{l}\text { Examinar comparativamente os efeitos de várias políticas governamentais avaliadas por um } \\
\text { conjunto de indicadores da economia digital a fim de descobrir se os seus efeitos são }\end{array}$ & Szeles e Simionescu, 2020 \\
\hline
\end{tabular}




\begin{tabular}{|c|c|}
\hline $\begin{array}{l}\text { positivos e consistentes entre os indicadores; comparação entre os novos e os antigos estados } \\
\text { membros da UE. }\end{array}$ & \\
\hline $\begin{array}{l}\text { Verificar se certas características dos países europeus são capazes de explicar o } \\
\text { relacionamento entre os cidadãos e o governo eletrônico e, também, os padrões de } \\
\text { comportamento e as formas que se relacionam com o e-governo. }\end{array}$ & $\begin{array}{l}\text { Pérez-morote e Pontones- } \\
\text { rosa, } 2020\end{array}$ \\
\hline $\begin{array}{l}\text { Examinar a relação entre TICs e a entrada da Internet com a performance educacional no } \\
\text { nível fundamental e médio na Malásia. }\end{array}$ & $\begin{array}{l}\text { Zuhairy, Tajuddin, Rohman, } \\
2018\end{array}$ \\
\hline $\begin{array}{l}\text { Avaliar os recursos potenciais dos territórios da Rússia, principalmente a desigualdade de } \\
\text { desenvolvimento entre as áreas urbanas e rurais, tendo como foco a desaceleração do } \\
\text { desenvolvimento das áreas periféricas. }\end{array}$ & Popova et. al., 2020 \\
\hline $\begin{array}{l}\text { Unindo a questão da exclusão digital feminina, buscaram entender se a inclusão das mulheres } \\
\text { no setor de TICS afeta o desenvolvimento sustentável e como esses fatores impactam nos } \\
\text { SDGs. }\end{array}$ & $\begin{array}{l}\text { Hayet-Kerras, Sánchez- } \\
\text { navarro, López-becerra, } \\
\text { Gómez, } 2020\end{array}$ \\
\hline $\begin{array}{l}\text { Entender os fatores que estão prejudicando a adoção dos serviços públicos digitais pelos } \\
\text { usuários através da análise das iniciativas de educação online. }\end{array}$ & Evans e Gomes, 2017 \\
\hline $\begin{array}{l}\text { Apresentar como a exclusão digital na saúde pode ser minimizada a partir da utilização das } \\
\text { bibliotecas como centros de inclusão digital. }\end{array}$ & Gann, 2019 \\
\hline $\begin{array}{l}\text { Definir os limiares para determinar a exclusão digital em zonas geográficas específicas e } \\
\text { com características similares para, com isso, poder analisar o fenômeno em questão. }\end{array}$ & $\begin{array}{l}\text { Mendoza-ruano e Caldera- } \\
\text { serrano, } 2014\end{array}$ \\
\hline $\begin{array}{l}\text { Explorar a interação entre idosos e organizações que prestam serviços públicos e } \\
\text { comunitários. }\end{array}$ & $\begin{array}{l}\text { Hodge, Carson, Carson, } \\
\text { Newman, Garrett, } 2017\end{array}$ \\
\hline
\end{tabular}

Fonte: Dados da Pesquisa

Afirmando as questões levantadas anteriormente, e corroborado com o levantamento de dados do estudo, pode-se dizer que a exclusão digital em processos de transformação digital reproduz desigualdades, como a de gênero (HAYET-KERRAS et. al, 2020) e problemas sociais já presentes, como a concentração de investimentos de infraestrutura e bem estar nas grandes cidades (PHILIP e WILLIAMS, 2019; MENDOZA-RUANO e CALDERA-SERRANO, 2014; ZEMTSOV, 2020, por exemplo). Dessa forma, fica claro que a tecnologia por si só não melhora a qualidade de vida ou que pode ser tida como sinônimo de desenvolvimento. Para que a transformação digital traga reais benefícios à sociedade e ao país, ela deve responder às necessidades reais da localidade, identificando-se, também, outros fatores necessários ao desenvolvimento sustentável, como justiça, igualdade de gênero e de oportunidades (ONU, 2020).

É interessante notar que, mesmo realizando a busca apenas com as palavras digital divide e digital transformation, os artigos analisados da RSL trouxeram a temática de responsabilidade governamental na criação de políticas públicas que mitigaram essa diferença. Pela estrutura dos artigos, ou eram resultados de políticas públicas implementadas em projetos de digitalização ou buscavam nos dados de surveys realizadas por entidades governamentais propostas para a criação de indicadores guias para confecção de políticas públicas. Mergel et.al. (2019) apresentam uma definição específica para a transformação digital no governo, que é:

A transformação digital é um esforço holístico para revisar os principais processos e serviços do governo, além dos esforços tradicionais de digitalização. Ele evolui ao longo de um contínuo de transição do analógico para o digital para uma revisão completa de políticas, processos atuais e necessidades do usuário e resulta em uma 
revisão completa dos existentes e na criação de novos serviços digitais. O resultado dos esforços de transformação digital enfoca, entre outros, a satisfação das necessidades dos usuários, novas formas de prestação de serviços e a expansão da base de usuários (p. 5).

Sendo assim, entende-se que há responsabilidade governamental em relação à acessibilidade às tecnologias e à infraestrutura digital necessária para conexão na rede. Não somente por um poder financeiro maior para obras de grande complexidade, mas também à mudança de postura em relação ao cidadão, buscando uma promoção de transparência e eficiência maior - não apenas no controle contábil, mas também na promoção de práticas mais democráticas de gestão. A TD na gestão pública pode causar mudanças tanto incrementais quanto radicais, sendo que, de qualquer forma, há a necessidade de flexibilidade organizacional para seguir com as mudanças (GONG, YANG, SHI, 2020), permitindo a formulação de políticas públicas que impactem na redução da exclusão digital.

De forma geral, pode-se constatar que os estudos de exclusão digital em processos de transformação digital ainda se preocupam com questões de indicadores macroeconômicos, sem avaliar sob a perspectiva indivíduo ou cidadão quanto ao acolhimento e receptividade das tecnologias - vide a baixa de estudos interpretativistas. Também, o recorte populacional para a análise ainda está limitado, podendo-se desagregar dados e expandir a pesquisa para outros grupos sociais minoritários ou que já sofrem outros processos de desigualdade social que vão influenciar no desenvolvimento (SEN, 2010).

Por fim, contribuindo para a o campo de estudos da exclusão digital, pode-se também identificar algumas expressões de exclusão digital que corroboram com a literatura atual, que são: a infraestrutura, acesso às TICs, a literacia digital (entendimento do uso e informações) e a eficácia (Bellini, 2018). Dentre estes, o principal ponto é a questão da eficácia, que seria uma etapa posterior à equidade digital dentro da sociedade digital e que une três eixos de atuação: limitação cognitiva, limitação de acesso e comportamental. Sendo assim, pode-se dizer que a área de Exclusão Digital em processos de TD possui um campo amplo ainda a ser explorado, principalmente quando entende-se que a exclusão digital trabalha processos sociotécnicos.

\section{CONSIDERAÇÕES FINAIS}

A sociedade passa por grandes mudanças ao longo do tempo. Atualmente, podemos citar a transformação digital como um vetor de transição a uma sociedade em rede (CASTELLS, 1999). Essa conexão se deu principalmente com a utilização das TICs, renovando nossas formas de interagir e produzir. Contudo, também evidenciou desigualdades já presentes em nosso mundo globalizado. Posto isto, este trabalho teve como objetivo identificar o que tem sido estudado sobre a exclusão digital dentro do contexto da transformação digital para explorer quais os principais temas já em pauta no campo acadêmico. Para isso, realizou-se uma Revisão Sistemática de Literatura em seis bases de dados, e a partir da análise dos 21 artigos selecionados, importantes achados puderam ser identificados.

Um primeiro ponto importante de destaque é a interdisciplinaridade do assunto. Os artigos selecionados são encontrados em periódicos das áreas rural, médica, econômica, administrativa, mostrando que este tema perpassa diferentes campos de conhecimento. Também, podemos identificar que este assunto é recente, pois não foi realizada uma definição limite de data e o artigo mais antigo identificado foi de 2014.

Outro aspecto importante está relacionado ao entendimento do que é exclusão digital. Mesmo os artigos utilizando formas diferenciadas de apresentar o conceito, foi possível identificar alguns pontos em comum que vão além do trinômio (sem) posse, (sem) acesso e (sem) conhecimento para utilizar. São eles: (i) falta de infraestrutura física para acessar a Internet, (ii) baixa escolaridade, (iii) diferença geracional, na qual as pessoas idosas possuem maior dificuldade de acesso e/ou utilizar as ferramentas. Também é perceptível que a exclusão digital pode ser analisada a partir de grupos sociais. Neste trabalho, foi identificada a questão da desigualdade de gênero (HAYETKERRAS et. al, 2020) e a diferença de infraestrutura digital das zonas rurais em relação às urbanizadas (POPOVA et. al., 2020). Pode-se somar a esses grupos, pessoas idosas com maior dificuldade de letramento tecnológico. Assim, é possível compreender que a exclusão digital é uma consequência negativa da era da Internet, talvez pela ausência de políticas públicas específicas na área, aprofundando as desigualdades já existentes em nossas sociedades, como aquelas indicadas pelo IDH. Pessoas mais pobres acabam sendo mais excluídas desse processo de desenvolvimento. 
Conforme apresentado anteriormente, muitos artigos relacionaram o poder público e exclusão digital. Sendo assim, é possível supor a responsabilidade da gestão pública em reduzir os efeitos dessa desigualdade, dado que é necessário o uso da capacidade estatal para reduzir as assimetrias, inclusive as de acesso e uso de TICs.

Também, é importante considerar o contexto de pandemia de COVID19 na sociedade em rede. Conforme apontado, a maioria dos estudos sobre exclusão digital estão registrados no ano de 2020. Em um mundo conectado e com a restrição de contato para conter a disseminação do vírus (AYRES, 2020), as TICs foram as principais ferramentas que possibilitam, por exemplo, o trabalho remoto e a comunicação entre pessoas, além de acesso a bens e serviços fundamentais. Conforme resultados da RSL, a exclusão digital está associdada a processos de TD. Sendo assim, uma consequência não-intencional surgida dessa rápida resposta que a pandemia exigiu de modernização, foi o aumento das desigualdades sociais em relação ao acesso e à posse de TICs, o qual foi duramente sentido pelas pessoas já excluídas socialmente por outros fatores como educação e renda (GOULARTI, 2020; PILL, 2020).

Outro aspecto a considerar são os incentivos e políticas públicas governamentais. Na medida em que os governos estabelecem iniciativas de transformação digital, estas precisam ser acompanhadas de políticas públicas de acesso à dispositivos de TICs, de acesso à Internet e de capacitação ao uso dos recursos. A distribuição do auxílio emergencial, por exemplo, trouxe à tona que as soluções de TD se baseiam em um público médio que não representa o cidadão brasileiro de forma ampla. Muitos cidadãos ficaram dias em filas tentando resolver o problema, e também gastaram muitos recursos em conexão $3 \mathrm{G} / 4 \mathrm{G}$, uma das mais caras do mundo (LUCIANO, 2020). Desta forma, as já conhecidas assimetrias econômicas se acentuam e se perpetuam.

De forma geral, o artigo contribuiu a partir da apresentação dos principais temas estudados sobre exclusão digital dentro da transformação digital. Para trabalhos futuros, seria importante traçar uma estrutura de estudo da exclusão digital para auxiliar nas pesquisas e na geração de conhecimento que auxilie na formulação de propostas que sejam capazes de alterar o status quo dessas desigualdades. Da mesma forma, cada local expressa uma característica de exclusão digital diferente, sendo importante a identificação das singularidades expressas por esse fenômeno derivado da transformação digital. Como limitação, identifica-se a impossibilidade de abranger todos os novos estudos na área por ser uma temática ainda em expansão, mas esta mesma restrição abre espaço para a continuidade deste trabalho de intensificar a busca pela ampliação deste conhecimento e, assim, enriquecer o campo de estudo de exclusão digital, o qual é importante socialmente e também, contemporâneo.

Neste sentido, a agenda de pesquisas pretende apresentar diferentes perspectivas e elementos relativos à exclusão digital e à transformação digital que poderiam ser abordados pela academia. Também, é uma forma de contribuir com temáticas para essa área de estudo que possui amplo espaço para debate e discussões. O Quadro 5 apresenta a proposta de agenda de pesquisa em questão.

Quadro 5 - Proposta de Agenda de Pesquisa

\begin{tabular}{|c|c|c|}
\hline Tema e Abordagem & $\begin{array}{l}\text { Posicionamento } \\
\text { Epistemológico }\end{array}$ & Implicações Metodológicas \\
\hline $\begin{array}{l}\text { Analisar indicadores e dados de Exclusão Digital e Transformação } \\
\text { Digital nos países da América Latina identificando as } \\
\text { problemáticas locais em comparação a outro grupo de países, } \\
\text { como a União Europeia. }\end{array}$ & Positivista & $\begin{array}{l}\text { Estudo quantitativo ou } \\
\text { quantitativo/qualitativo }\end{array}$ \\
\hline $\begin{array}{l}\text { Estudar a percepção dos cidadãos quanto às transformações } \\
\text { digitais proporcionadas pelo ente público para identificar a geração } \\
\text { de valor público pela inclusão digital }\end{array}$ & Interpretativista & $\begin{array}{c}\text { Estudo } \\
\text { qualitativo/quantitativo }\end{array}$ \\
\hline $\begin{array}{l}\text { Pesquisar a exclusão digital no contexto de processos de } \\
\text { transformação digital com recorte de renda e escolaridade } \\
\text { buscando conhecer a percepção dos usuários quanto ao uso das } \\
\text { TICs }\end{array}$ & Interpretativista & $\begin{array}{c}\text { Estudo } \\
\text { qualitativo/quantitativo }\end{array}$ \\
\hline Realizar análise comparativa sob a ótica do capitalismo & Positivista & Estudo de caso \\
\hline
\end{tabular}




\begin{tabular}{|l|l|l|}
\hline $\begin{array}{l}\text { dependente, identificando os processos de transformação digital } \\
\text { entre os países ou regiões e suas diferenças e como isso pode gerar } \\
\text { exclusão digital ou acentuá-la }\end{array}$ & Positivista \\
\hline $\begin{array}{l}\text { Investigar a existência de ações de inclusão digital para o público } \\
\text { idoso em processos de transformação digital quanto a sua } \\
\text { efetividade e qualidade de vida }\end{array}$ & quantitativo/qualitativo \\
\hline $\begin{array}{l}\text { Compreender como os fatores locais de determinadas regiões } \\
\text { influenciam inclusão ou exclusão em processo de transformação } \\
\text { digital }\end{array}$ & Positivista & quantitativo/qualitativo \\
\hline $\begin{array}{l}\text { Compreender o quanto a exclusão digital reduz ou impede o } \\
\text { exercício pleno da cidadania para populações já pertencentes a } \\
\text { outros grupos e processos de desigualdade social }\end{array}$ & Interpretativista & Estudo de caso \\
\hline
\end{tabular}

Fonte: Elaborado pelos autores.

Dentro do exposto no Quadro 5, sugere-se a avaliação da efetividade de ações de inclusão digital quanto à literacia digital (segunda forma de exclusão digital), tendo em vista de que o entendimento que a falta de acesso é uma exclusão primária. Também, é importante incentivar o estudo desse fenômeno na América Latina, levandose em consideração as peculiaridades que o desenvolvimento dessa região possui em relação às demais localidades estudadas nos artigos selecionados. Juntamente, recomenda-se propor processos de inclusão digital que viabilizem a diminuição das desigualdades em qualquer nível encontrado - por localidade, idade, gênero, raça, renda. Como proposta de trabalho do nosso grupo de pesquisa, buscar-se-á entender o processo de desenvolvimento da transformação digital de forma que a exclusão seja mitigada no design do projeto, principalmente a partir da percepção do cidadão, que é o principal ator impactado, buscando a geração de valor público e bem-estar social.

No início da informatização, no começo dos anos 90, a inclusão digital representava a possibilidade de utilizar um editor de texto ou planilha, ou inserir informação, fazer consultas e emitir relatório em Sistemas de Informação. Pouco depois, nos anos e-mail, envolvia também a utilização de browsers para buscas na Internet e o envio de e-mail. Até aqui, o foco era em ser mais competitivo na busca por um emprego. Atualmente, a inclusão digital é social e é política. Seu papel não é apenas para comunicação entre indivíduos, realização de atividades professionais e de lazer, compra ou venda de bens, mas é fundamental ao exercício de cidadania. O exercício da cidadania implica em acesso e compressão a informações do governo, participação, transparência, entendimento de quando uma informação tem indícios de não ser verdadeira, interação com os governos - todas estas ações demandam um cidadão incluído digitalmente e com condições de fazer as suas próprias escolhas, o que reforça a importância de que os processos de transformação digital não sejam (ainda mais) excludentes.

Notas: O presente trabalho foi realizado com apoio da Coordenação de aperfeiçoamento de Pessoal de Nível Superor - Brasil (CAPES) - Código de Financiamento 001.

\section{REFERÊNCIAS}

ALEKSANDROVNA, M. O., IURIEVNA, E. M., OLEGOVNA, E. P. Digital Transformation as The Factor of The Generation Dynamics in The Information Society. QUID 2017, pp. 1624-1629, Special Issue $N^{\circ} 1$ - ISSN: 1692-343X, 2017.

ASGARKHANI, M. Digital government and its effectiveness in public management reform: A local government perspective. Public Management Review, 7(3), p. 465-487, 2005.

AYRES, J. S. A metabolic handbook for the COVID-19 pandemic. Nature metabolism, vol 2, p. 572-585, jul. 2020. BELLINI, C. G. P. The ABCs of effectiveness in the digital society. Communications of the ACM, Jun. 2018 
https://doi.org/10.1145/3205945

BRERETON, P., KITCHENHAM, B. A., BUDGEN, D., TURNER, M., KHALIL, M. Lessons from applying the systematic literature review process within the software engineering domain. The Journal of Systems and Software, 80, p. 571-583 2007.

BOK, J., PORUMBESCU, G. A. Engendering inclusive e-government use through citizen IT training programs. Government Information Quarterly, 36(1), p. 69-76, 2019.

CASTELLS, M. A. Sociedade em Rede - A era da informação: economia, sociedade e cultura; v.1). Tradução Roneide Venâncio Majer. São Paulo: Paz e Terra, 1999.

CHETTY, K., QIGUI, L., GCORA, N., JOSIE, J., WENWEI, L., FANG, C. Bridging the digital divide: measuring digital literacy. Economics: The Open-Access, Open-Assessment E-Journal, 12 (23): 1-20, 2018 http:// dx.doi.org/10.5018/economics-ejournal.ja.2018-23.

CORDELLA, A., TEMPINI, N. E-government and organizational change: Reappraising the role of ICT and bureaucracy in public service delivery. Government Information Quarterly, 32, p. 279-286, 2015.

CURTIS, S. Digital transformation-the silver bullet to public service improvement? Public Money \& Management, 39 (5), p. 322-324, 2019, DOI:10.1080/09540962.2019.1611233.

DOMÍNGUEZ, M. M. Acceso y uso de tecnologías de la información y comunicación en México: factores determinantes. Revista de Tecnología y Sociedad, 8 (14), p. 1-18, 2018.

EVANS, A. M., GOMES, R. G. The Role of Digital Literacy in Citizens' Adoption of Digital Public Services: the Portuguese Case. European Conference on Digital Government, 2017.

GANN, B. Transforming lives: Combating digital health inequality. IFLA Journal, 45(3), p. 187-198, 2019.

GUILFOY, V., MURRAY, S., OWEN, E. [orgs.] ICT Literacy Panel. Digital Transformation: A Framework for ICT Literacy. A Report of the International ICT Literacy Panel. Educational Testing, 2002.

GONG, Y., YANG, J., SHI, X. Towards a comprehensive understanding of digital transformation in government: Analysis of flexibility and enterprise architecture. Government Information Quarterly, 37, 101487, 2020. DOI https://doi.org/10.1016/j.giq.2020.101487

GOULARTI, J. G. A escalada da desigualdade em meio à “coronacrise”. Le Monde Diplomatique, Lições das crises econômicas, Edição 155, jun. 2020 Disponível em: https://diplomatique.org.br/a-escalada-dadesigualdade-em-meio-a-coronacrise/

HAYET KERRAS, H., SÁNCHEZ-NAVARRO, J. L., LÓPEZ-BECERRA, E. I., GÓMEZ, M. D. M. The Impact of the Gender Digital Divide on Sustainable Development: Comparative Analysis between the European Union and the Maghreb. Sustainability, 12, p. 1-30, 2020.

HODGE, H., CARSON, D., CARSON, D., NEWMAN, L., GARRETT, J. Using Internet technologies in rural communities to access services: The views of older people and service providers. Journal of Rural Studies, 54, p. 469-478 2017.

HOSMAN, L., COMISSO, M. A. P. How do we understand "meaningful use" of the internet? Of divides, skills and socio-technical awareness. Journal of Information, Communication and Ethics in Society, 18 (3), p. 461479, 2020. https://doi.org/10.1108/JICES-05-2020-0055

IBGE. Pesquisa Nacional Por Amostra De Domicílios Contínua - Pnad Contínua: Acesso À Internet E À Televisão E Posse De Telefone Móvel Celular Para Uso Pessoal. PNAD CONTÍNUA, 2018.

KUPRIYANOVA, M., DRONOV, V., GORDOVA, T. Digital Divide of Rural Territories in Russia. Agris on-line Papers in Economics and Informatics, XI (3), p. 85-90, 2019.

LATAPU, P. P., LUTUI, R., VAIPUNA, S., MOALA, K., FONUA, S., LIU, W., GUTIERREZ, A., SONG, J., CHIARAVIGLIO, L., WU, J., BAGULA, A. Bridging the Digital Divide In Tonga Through A Sustainable Multi-tenancy Broadband Infrastructure: Are We Ready? IEEE International Conference, p. 1-6, 2018.

LUCIANO, E. M. Information management hits and misses in the COVID19 emergency in Brazil. International Journal of Information Management, 55, 102194, 2020.

LUCIANO, E. M. Exclusão digital e responsabilidade social: proposta de um framework de análise. In: XVII Congresso da SLADE - Sociedad Latinoamericana de Estratégia, 2004, Itapema. Anais do XVII SLADE, 
2004.

MENDOZA-RUANO, J. J., CALDERA-SERRANO, J. Umbrales para la determinación de la brecha digital: Comparativa entre regiones desarrolladas. TransInformacao, 26(2), p. 125-132, 2014.

MERGEL, I., EDELMANN, N., HAUG, N. Defining digital transformation: Results from expert interviews. Government Information Quarterly, 36 (4), 1-16, 2019.

MENDES-DA-SILVA, W. Contribuições e limitações de revisões narrativas e sistemáticas na área de negócios. Revista de Administração Contemporânea, 23(2), 1-11, 2019. https://doi.org/10.1590/19827849rac2019190094

MINISTÉRIO DA CIDADANIA. Auxílio emergencial: tutorial de acesso. 2020 Disponível em: https://www.gov.br/cidadania/pt-br/servicos/auxilio-emergencial. Acesso em 19 de out de 2020.

Objetivos de Desenvolvimento Sustentável. ONU. Disponível em: https://brasil.un.org/pt-br/sdgs/ Acesso em: 2 de nov. de 2020

OECD. "Understanding the Digital Divide". OECD Digital Economy Papers, No. 49, OECD Publishing, Paris, 2001. http://dx.doi.org/10.1787/236405667766

OTIOMA, C., MAFALDA, A., MARTINEZ, J. Spatial analysis of urban digital divide in Kigali, Rwanda. GeoJournal, 84(3), p. 719-741, 2019.

PÉREZ-MOROTE, R., PONTONES-ROSA, C. Technological Forecasting \& Social Change The effects of egovernment evaluation, trust and the digital divide in the levels of e-government use in European countries. Technological Forecasting \& Social Change, 154. https://doi.org/10.1016/j.techfore.2020.119973, 2020

PILL, D. Educação na pandemia deve priorizar reflexão e cidadania, dizem experts. UOL, Ecoa, Educação, jun, 2020. Disponível em: https://www.uol.com.br/ecoa/ultimas-noticias/2020/06/13/educacao-na-pandemiadeve-priorizar-reflexao-e-cidadania-dizem-experts.htm

RÄISÄNEN, J., TUOVINEN, T. Digital innovations in rural micro-enterprises. Journal of Rural Studies, 73, p. $56-67,2020$

REIS, J., AMORIM, M., MELAO, N., MATOS, P. Digital Transformation: A Literature Review and Guidelines for Future Research. 10th European Conference on Information Systems Management. Academic Conferences and publishing limited, 2016.

SCHALlMO, D., WILliAMNS, C. A., Boardman, L. Digital Transformation Of Business Models - Best Practice, Enablers, And Roadmap. International Journal of Innovation Management, 21 (8), 1740014, 2017 DOI: $10.1142 / \mathrm{S} 136391961740014 \mathrm{X}$

SCHWAB, K. A quarta revolução industrial (1st ed.). São Paulo: Edipro, 2016.

SEN, A. Desenvolvimento como liberdade. Tradução: Laura teixeira Motta. São Paulo: Companhia das Letras, 2010.

SERVON, L. J. Bridging the Digital Divide: Technology, Community, and Public Policy. USA: Blackwell Publishing, 2013.

SZELES, M. R., SIMIONESCU, M. Regional Patterns and Drivers of the EU Digital Economy. Social Indicators Research, 2020. https://doi.org/10.1007/s11205-020-02287-x

VASILESCU, M. D., SERBAN, A. C., DIMIAN, G. C., ID, I. A., PICATOSTE, X. Digital divide, skills and perceptions on digitalisation in the European Union- Towards a smart labour market. Plos One, p. 1-39, 2020. https://doi.org/10.1371/journal.pone.0232032

VIAL, G. Understanding digital transformation: A review and a research agenda. Journal of Strategic Information Systems, 28, 118-144, 2019.

MIKLIAN, J., HOELSCHER, K. Smart Cities, Mobile Technologies and Social Cohesion in India. Indian Journal of Human Development, 11(1), p. 1-16, 2017.

VIALE PEREIRA, G., ESTEVEZ, E., CARDONA, D., CHESÑEVAR, C., COLLAZZO-YELPO, P., \& SCHOLZ, R. South American expert roundtable: increasing adaptive governance capacity for coping with unintended side effects of digital transformation. Sustainability, 12(2), 718, 2020.

VIÑARÁS-ABAD, M., ABAD-ALCALÁ, L., LLORENTE-BARROSO, C., SÁNCHEZ-VALLE, M., PRETEL- 
JIMÉNEZ, M. e-Administration and the e- inclusion of the elderly. Revista Latina de Comunicación Social, 72, pp. 197-219, 2017.

PHILIP, L., WILLIAMS, F. Remote rural homebased businesses and digital inequalities: Understanding needs and expectations in a digitally underserved community. Journal of Rural Studies, 68, p. 306-318, 2019.

POPOVAET. AL. The impact of the digital divide on the development of socio-economic systems. IOP Conference Series: Earth and Environmental Science, 2020.

ZEMTSOV, S. New technologies, potential unemployment and 'nescience economy' during and after the 2020 economic crisis. Regional Science Policy \& Practice, doi: 10.1111/rsp3.12286, 2020.

ZUHAIRY, M., TAJUDDIN, M., ROHMAN, I. The Impact of Broadband Penetration on the Student Performance in Primary and Secondary School in Malaysia. ICEGOV '18, April, p. 111-117, 2018. 